\title{
GESTIÓN DE INTERNACIONALIZACIÓN DE LOS PROGRAMAS DE POST GRADO EN CIENCIAS ECONÓMICAS Y EMPRESARIALES
}

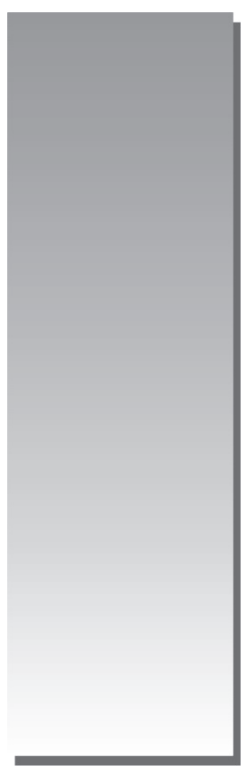

Juan Victoriano Castillo Maza*

E-mail: juan5705@yahoo.es

\begin{abstract}
RESUMEN
En el marco del proceso de internacionalización promovido por la Universidad Nacional Mayor de San Marcos (UNMSM), las Unidades de Post Grado de las Facultades de Ciencias Económicas y Ciencias Administrativas de la UNMSM, al amparo del Convenio de Cooperación Interinstitucional con la Universidad Montesquieu Bordeaux IV de Francia con la experiencia de casi dos décadas de prestación de servicios de perfeccionamiento del más alto nivel académico en los programas de Maestría y Doctorado, vienen ofreciendo en los últimos cinco años maestrías y doctorados internacionales de doble grado, con resultados exitosos.

La Unidad de Post Grado de la Facultad de Ciencias Económicas ofrece la Maestría y el Doctorado en Ciencias de Gestión, así como la Facultad de Ciencias Administrativas ofrece el programa Master en Management International otorgado por el Instituto de Administración de Empresas de la Universidad Montesquieu-Bordeaux IV de Francia y la Maestría Profesional en Gestión de Negocios Internacionales con mención en Comercio Internacional o Finanzas Internacionales, otorgado por la Unidad de Posgrado de la Facultad de Ciencias Administrativas de la Universidad Nacional Mayor de San Marcos (Lima - Perú).
\end{abstract}

Palabras clave: Post Grado internacional doble grado.

\section{ABSTRACT}

As part of the internationalization process promoted by the Universidad Nacional Mayor de San Marcos (San Marcos University), Units of Graduate Schools of Economics and Administrative Sciences under Interinstitutional Cooperation Agreement with the University Montesquieu Bordeaux IV of France and Universidad Nacional Mayor de San Marcos, with the experience of nearly two decades of improving service delivery of the highest academic level in the Masters and Doctoral programs, are offered in the past five years international masters and doctoral dual degree, with results successful.

* Doctor en Ciencias Contables y Empresariales, Magister en Economía con mención en métodos Cuantitativos por la UNMSM. Licenciado en Administración por la Universidad Nacional de Trujillo. Profesor Principal de la Facultad de Ciencias Administrativas de la UNMSM. Profesor de Postgrado de universidades nacionales y privadas: UNMSM, UNFV, UNASAM, UNSAAC, UNJBG, UNP, UNCP, UNA, UCV y UANCV. 
The Faculty of Economics offers a Masters and PhD in Management Sciences and the Faculty of Management offers the Master program in International Management awarded by the Institute of Business Administration from the University Montesquieu-Bordeaux IV of France and the Masters Training in International Management with a concentration in International Trade or International Finance Unit given by the Graduate School of Management at the Universidad Nacional Mayor de San Marcos (Lima - Peru).

Key words: International Management Masters and PhD Programs.

\section{INTRODUCCIÓN}

En un contexto de globalización caracterizado por un progresivo acercamiento entre los agentes que intervienen en la vida económica de los países (gobiernos, empresas y consumidores o usuarios), una competencia cada vez más intensa y mayores oportunidades de expansión internacional, una opción estratégica básica para las empresas es invertir en sus procesos de internacionalización.

La internacionalización de las empresas en general y de la empresa peruana en particular es una realidad cada vez más sólida y es consecuencia del desarrollo de sus recursos y capacidades, y del aprovechamiento de las oportunidades de negocios que ofrecen los mercados mundiales. La planificación y gestión óptima de este fenómeno requiere, entre dichos recursos y capacidades, de personal especializado con formación en áreas de gestión de comercio exterior y finanzas internacionales.

En armonía con el proceso de internacionalización y en aplicación del convenio de cooperación suscrito y aprobado entre la Universidad Montesquieu-Bordeaux IV de Francia y la Universidad Nacional Mayor de San Marcos de Perú, la Facultad de Ciencias Económicas desde hace cinco años y la Facultad de Ciencias Administrativas hace tres años, a través de sus Unidades de Post Grado, ofrecen programas de perfeccionamiento internacional de doble grado: la Maestría en Ciencias de la Gestión Económico Empresarial y el Doctorado en Gestión Económica Global; y la Maestría Profesional de Gestión de Negocios Internacionales con mención en Comercio Internacional y Finanzas Internacionales, respectivamente.

\section{HIPÓTESIS}

Una gestión eficiente de la internacionalización de los programas de perfeccionamiento en el área de las Ciencias Económicas y Empresariales reforzará la competitividad de los profesionales que accedan a dichos programas.

\section{MÉTODOS Y RESULTADOS}

La Unidad de Post Grado de la Facultad de Ciencias Económicas de la Universidad Nacional Mayor de San Marcos ofrece la Maestría en Ciencias de la Gestión Económico Empresarial cuyo objetivo principal es desarrollar criterios para la toma de decisiones empresariales. En ese sentido, busca integrar conocimientos teóricos con experiencias prácticas de las más variadas instituciones, a través del estudio y análisis de casos, y de la interacción con ejecutivos líderes en el mundo de los negocios. Al concluir los estudios, el Magíster de este programa estará en condiciones de:

- Asumir el liderazgo en la toma de decisiones en los distintos niveles gerenciales de la empresa, diseñando e implementando estrategias de negocios para optimizar la ventaja competitiva de la organización, y creando valor mediante el uso de las últimas técnicas y herramientas.

- Dirigir, administrar y controlar la marcha de organizaciones; es decir, realizar su gestión empresarial.

- Motivar la gestión económica que oriente las acciones con gran eficiencia y eficacia en las organizaciones.

Al finalizar satisfactoriamente los estudios y luego de la aprobación de la Tesis de Grado, los egresados reciben el doble grado:

- Magíster Académico en Ciencias de la Gestión Económico Empresarial, otorgado por la Facultad de Ciencias Económicas de la Universidad Nacional Mayor de San Marcos.

- Máster en Gestion a finalite Recherche, Mention Management, Specialité RECHERCHE EN GESTION DES ORGANISATIONS otorgado por la Universidad de Montesquieu Bordeaux IV, de Francia.

La Maestría en Ciencias de Gestión Económico Empresarial es pre-requisito para postular al Doctorado en Gestión Económica Global y Ciencias de 
la Gestión, con doble grado académico (UNMSM y Universidad de Montesquieu Bordeaux IV).

Las ciencias económicas han logrado un importante desarrollo a nivel global, por lo que resulta necesario contar con profesionales que, analizando los acontecimientos, planteen las soluciones más adecuadas pero que a la vez investiguen las razones y consecuencias de determinados comportamientos económicos, apoyando así el desarrollo de la ciencia económica. Es por ello que la Unidad de Post Grado de la Facultad de Ciencias Económicas de la Universidad Nacional Mayor de San Marcos desarrolla el Doctorado en Gestión Económica Global, programa de estudios que permite que sus egresados obtengan una doble titulación otorgada por la UNMSM y por la Universidad Montesquieu Bordeaux IV.

Al concluir sus estudios, el egresado del doctorado estará en condiciones de:

- Promover permanentemente las investigaciones en Ciencias de la Gestión y Gestión Económica Global.

- Aplicar los conocimientos adquiridos de la gestión empresarial (conceptos, métodos y herramientas) y analizar su desempeño y resultados obtenidos, para crear modelos adecuados a nuestra realidad que permitan lograr el desarrollo de la economía y la administración.

- Formar docentes investigadores que contribuyan al planteamiento de alternativas de solución para los problemas que se presenten a nivel nacional, regional y local.

La Unidad de Post Grado de la Facultad de Ciencias Administrativas de la UNMSM ofrece el programa internacional de doble grado de Máster en Management International por el Instituto de Administración de Empresas de la Universidad Montesquieu Bordeaux IV de Francia y el programa de Maestría Profesional de Gestión de Negocios Internacionales con mención en Comercio Internacional y Finanzas Internacionales por la Universidad Nacional Mayor de San Marcos de Perú, dirigido a profesionales graduados en diferentes áreas académico-profesionales vinculados a los negocios y a las ciencias empresariales, quienes, al finalizar sus estudios, estarán en condiciones de:

- Asumir el liderazgo en la toma de decisiones en los diferentes niveles gerenciales de las empresas y sectores de la economía como servicios, bancario, seguros y finanzas internacionales, con el fin de diseñar e implementar estrategias de negocios y gestión sobre la base de optimizar las ventajas competitivas de la organización donde participan.

- Planear, organizar, dirigir, integrar y fiscalizar la marcha de las empresas y organizaciones internacionales y globales.

- Tomar decisiones en los diferentes aspectos de la gestión económica y empresarial sobre la base de una visión integral de las organizaciones de gestión política y de los niveles de decisión.

- Estimular la participación y compromiso de cada uno de los miembros de la organización para lograr el éxito gracias a un buen desempeño en las empresas donde laboran.

- Elaborar planes de comercio internacional e investigar el mercado nacional e internacional mediante la búsqueda sistemática de información en diversas fuentes.

- Analizar los entornos internacionales de las empresas para detectar tendencias de desarrollo e identificar las amenazas y oportunidades.

- Analizar y seleccionar mercados con una metodología específica, utilizando criterios tales como atractivo de mercado, ventaja competitiva y el riesgo.

Al finalizar el programa se obtiene el doble grado académico simultáneo:

- Título de Master en Management International otorgado por la Universidad Montesquieu Bordeaux IV de Francia.

- Grado de Magister en Gestión de Negocios Internacionales con mención en Comercio Internacional o Finanzas Internacionales por la Universidad Nacional Mayor de San Marcos de Perú.

\section{ANÁLISIS Y DISCUSIÓN}

Los resultados obtenidos a la fecha por la Unidad de Post Grado de la Facultad de Ciencias Administrativas durante los tres años de vigencia del convenio específico se muestran a continuación:

\section{Admisión 2008-I:}

$\begin{array}{ll}\text { Ingresantes } & 15 \\ \text { Matriculados } & 13\end{array}$


Egresados

Graduados (*)

13

13

(*)Títulos otorgados por la Universidad Montesquieu Bordeaux IV, que fueron entregados por el señor Rector de la UNMSM, doctor Luis Fernando Izquierdo Vásquez, en ceremonia especial realizado el pasado mes de octubre en la sala de sesiones del rectorado.

Al finalizar esta primera promoción podemos afirmar categóricamente la eficacia del programa, con un $100 \%$ de matriculados, egresados y graduados por parte de la Universidad Montesquieu Bordeaux IV Francia.

\section{Admisión 2009-I:}

$\begin{array}{ll}\text { Ingresantes } & 12 \\ \text { Matriculados } & 10\end{array}$

Estos alumnos actualmente se encuentran culminando los estudios en el semestre 2010-II. La tendencia eficaz del programa se mantiene en esta segunda promoción, al mantener los mismos resultados en los cuatro semestres de estudio.

\section{Admisión 2010-I:}

$\begin{array}{ll}\text { Ingresantes } & 20 \\ \text { Matriculados } & 18\end{array}$

Actualmente se encuentran cursando el segundo ciclo de estudios y deben culminar en el semestre académico 2011-II.

\section{CONCLUSIONES}

- El desarrollo de programas de post grado internacional de doble grado se realiza en colaboración y cooperación con instituciones educativas en un mundo globalizado.

- La gestión eficaz de la internacionalización de programas de post grado a través de convenios de cooperación interinstitucional, es decir el trabajo colaborativo, permite aprovechar las fortalezas y oportunidades y superar las debilidades y amenazas del entorno.

- Los indicadores de la titulación de los egresados por parte de la Universidad de Montesquieu Bordeaux IV de Francia se encuentra en niveles de 80 y $100 \%$.

\section{BIBLIOGRAFÍA}

\section{Convenios Marco:}

Convenio de Cooperación suscrito entre la Universidad Montesquieu-Bordeaux IV de Francia y la Universidad Nacional Mayor de San Marcos de Perú, aprobado por Resolución Rectoral №04814R-03 de fecha 09 de septiembre de 2003, con vigencia de cinco años, firmado por el doctor Gerard Hirigoyen, Presidente de la Universidad Montesquieu-Bordeaux IV, y el doctor Juan Manuel Burga Díaz, Rector de la Universidad Nacional Mayor de San Marcos.

Convenio de Cooperación entre la Universidad Montesquieu Bordeaux IV de Francia y la Universidad Nacional Mayor de San Marcos, de Perú, con vigencia de cinco años, firmado por el doctor Lean-Pierre Laborde, Presidente de la Universidad Montesquieu Bordeaux IV y el doctor Luis Fernando Izquierdo Vásquez, Rector de la Universidad Nacional Mayor de San Marcos.

\section{Convenios específicos:}

Acuerdo de Gestión Interinstitucional suscrito entre la Facultad de Ciencias Administrativas de la Universidad Nacional Mayor de San Marcos de Perú y el Instituto de Administración de Empresas de la Universidad Bordeaux IV de Francia, aprobado por Resolución Rectoral № 01361-R-08 de fecha 28 de marzo de 2008, con vigencia de tres años a partir del 16 de octubre del 2007.

Convenio Específico de Cooperación Interinstitucional entre el Instituto de Administración de Empresas de la Universidad Montesquieu Bordeaux IV, aprobado por resolución Rectoral № 04429-R-10 de fecha 20 de agosto del 2010, firmado por M. Jean-Pierre Laborde, Presidente de la Universite Montesquieu Bordeaux IV, y M. Serge Evraert, Director del Institut d'e Administration des Entreprises, y la Facultad de Ciencias Administrativas de la Universidad Nacional Mayor de San Marcos, firmados por el Rector de la UNMSM, doctor Luis Fernando Izquierdo Vásquez, con vigencia de dos años. 